\title{
Synthesis of Seven-Membered Carbocyclic Rings via Microwave-Assisted Tandem Oxyanionic 5-Exo Dig Cyclization-Claisen Rearrangement Process
}

Xin Li, Robert E. Kyne and Timo V. Ovaska*

Connecticut College, Department of Chemistry, 270 Mohegan Avenue, New London, CT 06320, USA

tvova@conncoll.edu

\section{Supporting Information}

\section{Contents:}

General Experimental

Experimental Procedures page S2

pages S2-S11 


\section{General Experimental.}

${ }^{1} \mathrm{H}$ and ${ }^{13} \mathrm{C}$ NMR spectra were recorded in $\mathrm{CDCl}_{3}$. Chemical shifts are reported in units of parts per million (ppm), relative to tetramethylsilane at $\delta=0.00 \mathrm{ppm}$. Coupling constants $(J)$ are reported in hertz (Hz). Infrared spectra are reported in $\mathrm{cm}^{-1}$.

All microwave experiments were conducted in a CEM Focused Microwave ${ }^{\mathrm{TM}}$ Synthesis System, Model Discover microwave oven, equipped with an infrared temperature control system. All microwave reactions were performed in sealed $10 \mathrm{~mL}$ microwave vials.

Tetrahydrofuran (THF) was freshly distilled under $\mathrm{N}_{2}$ from dark blue solutions of sodium benzophenone ketyl. Phenetole (PhOEt), $\mathrm{CH}_{2} \mathrm{Cl}_{2}$ (DCM), TMSCl, and $\mathrm{Et}_{3} \mathrm{~N}$ were freshly distilled from calcium hydride. The concentrations of solutions of organolithium reagents were determined by titrations with sec-butyl alcohol using 1,10-phenantroline as the indicator following the method of Watson and Eastham. ${ }^{1}$ All glassware was flame-dried under an inert atmosphere and all reactions were performed under an atmosphere of dry argon or nitrogen.

\section{Experimental Procedures}

\section{5-(2,5-Dimethoxyphenyl)pent-4-ynal (1)}

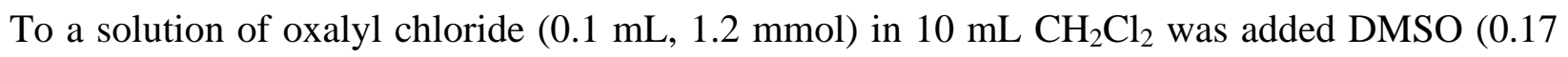
$\mathrm{ml}, 2.4 \mathrm{mmol}$ ) at $-78^{\circ} \mathrm{C}$ and the resulting mixture was stirred for $20 \mathrm{~min}$. To this was then added 5-(2,5-dimethoxyphenyl)pent-4-yn-1-ol (106 mg, $0.48 \mathrm{mmol}$ ) in $3 \mathrm{~mL} \mathrm{CH}_{2} \mathrm{Cl}_{2}$ at $-78^{\circ} \mathrm{C}$. After $50 \mathrm{~min}$ of further stirring at this temperature, $\mathrm{NEt}_{3}$ (486 mg, $4.8 \mathrm{mmol}$ ) was added and the reaction mixture was allowed to warm to room temperature. The mixture was then poured into saturated $\mathrm{NaHCO}_{3}$ solution, the layers were separated and the aqueous layer was extracted with EtOAc. The combined organic extracts were washed with brine, dried over $\mathrm{MgSO}_{4}$, filtered, and concentrated under reduced pressure. The crude product was purified by column chromatography (20\% EtOAc in hexane) to give aldehyde 1 as yellow solid (99 mg, 95\%). mp: 63을 2924, 1716, 1496, 1039, 821; ${ }^{1} \mathrm{H}$ NMR $\left(\mathrm{CDCl}_{3}, 500 \mathrm{MHz}\right) \delta 9.84$ (s, $1 \mathrm{H}$ ), 6.90 (d, $J=3.0 \mathrm{~Hz}, 1$ H), 6.81-6.75 (m, 2 H), 3.81 (s, $3 \mathrm{H}$ ), 3.74 (s, $3 \mathrm{H}), 2.78-2.77$ (m, $4 \mathrm{H})$; ${ }^{13} \mathrm{C}$ NMR $\left(\mathrm{CDCl}_{3}, 125\right.$ MHz) $\delta$ 200.6, 154.3, 153.1, 118.3, 115.1, 112.9, 111.8, 91.8, 77.6, 56.3, 55.7, 42.6, 13.0; HRMS (EI) calcd for $\mathrm{C}_{13} \mathrm{H}_{14} \mathrm{O}_{3}\left(\mathrm{M}^{+}\right) \mathrm{m} / \mathrm{z} 218.0943$, found 218.0940.

\footnotetext{
${ }^{1}$ S. C. Watson, J. F. Eastham, J. Organomet. Chem. 1967, 9, 165-168.
} 
1-Cyclopentenyl-5-(2,5-dimethoxyphenyl)pent-4-yn-1-ol (2). To a $-78^{\circ} \mathrm{C}$ solution of 1iodocyclopentene (776 mg, $4.00 \mathrm{mmol}$ ) in $15 \mathrm{~mL} \mathrm{Et}_{2} \mathrm{O}$ was added ${ }^{t} \mathrm{BuLi}$ (1.7 $\mathrm{M}$ in pentane, 4.7 $\mathrm{mL}, 8.0 \mathrm{mmol}$ ) dropwise. The resulting solution was stirred at $-78^{\circ} \mathrm{C}$ for $15 \mathrm{~min}$, then warmed to $0{ }^{\circ} \mathrm{C}$ and stirred for another $15 \mathrm{~min}$ at this temperature to destroy any excess ${ }^{t} \mathrm{BuLi}$. The solution was then cooled to $-78{ }^{\circ} \mathrm{C}$, followed by the dropwise addition of aldehyde 1 (436 mg, $2.00 \mathrm{mmol})$ dissolved in $\mathrm{Et}_{2} \mathrm{O}(15 \mathrm{~mL})$. The reaction mixture was stirred thereafter for $20 \mathrm{~min}$ and then quenched by the addition of aqueous $\mathrm{NH}_{4} \mathrm{Cl}$. The layers were separated and the aqueous layer was extracted with $\mathrm{Et}_{2} \mathrm{O}$. The combined organic extracts were washed with brine, dried over $\mathrm{MgSO}_{4}$, filtered, and concentrated under reduced pressure. The crude product was purified by column chromatography (20\% EtOAc in hexane) to give alcohol 2 as a pale yellow oil (509 mg, 89\%). IR (neat) 3446, 2927, 1605, 1495, 1049, $824 \mathrm{~cm}^{-1} ;{ }^{1} \mathrm{H} \mathrm{NMR}\left(\mathrm{CDCl}_{3}, 500 \mathrm{MHz}\right) \delta$ 6.89 (d, $J=3.0 \mathrm{~Hz}, 1 \mathrm{H}), 6.76-6.72$ (m, $2 \mathrm{H}$ ), 5.63 (d, $J=1.5 \mathrm{~Hz}, 1 \mathrm{H}), 4.45$ (dd, $J=6.0,4.5 \mathrm{~Hz}$, 1 H), 3.79 (s, 3 H), 3.71 (s, 3 H), 2.58-2.46 (m, 2 H), 2.44 (br s, 1 H), 2.32-2.27 (m, 4 H), 1.921.80 (m, $4 \mathrm{H}) ;{ }^{13} \mathrm{C} \mathrm{NMR}\left(\mathrm{CDCl}_{3}, 125 \mathrm{MHz}\right) \delta 154.2$, 153.0, 146.3, 125.4, 118.0, 114.6, 113.2, 111.6, 93.9, 77.1, 70.2, 56.1, 55.5, 34.1, 32.1, 31.2, 23.2, 16.0; HRMS (EI) calcd for $\mathrm{C}_{18} \mathrm{H}_{22} \mathrm{O}_{3}$ $\left(\mathrm{M}^{+}\right) \mathrm{m} / \mathrm{z}$ 286.1569, found 286.1569.

\section{(3aS*,4R*)-4-(2,5-Dimethoxyphenyl)-2,3,3a,4,6,7-hexahydroazulen-5(1H)-one (3)}

\section{General procedure for the microwave-assisted cyclization/Claisen rearrangement:}

Alcohol 2 (100 mg, $0.35 \mathrm{mmol})$ was transferred to a $10 \mathrm{~mL}$ flame dried microwave vial along with anhydrous phenetole $(1.5 \mathrm{~mL})$. A ca. $10 \mathrm{~mol} \% \mathrm{MeLi}$ in $\mathrm{Et}_{2} \mathrm{O}$ was added and the solution was heated at $210^{\circ} \mathrm{C}$ for $45 \mathrm{~min}$ in the microwave oven. The phenetole solvent was then removed in vacuo and the residue was directly purified by column chromatography (5\% to 10\% EtOAc in hexane) to give ketone 3 as a pale yellow oil (76mg, 76\%).

IR (neat) 2936, 1705, 1496, 1225, $823 \mathrm{~cm}^{-1}$; ${ }^{1} \mathrm{H} \mathrm{NMR}\left(\mathrm{CDCl}_{3}, 500 \mathrm{MHz}\right) \delta$ 6.77- $6.69(\mathrm{~m}, 3 \mathrm{H})$, 5.56 (s, 1 H), 3.76 (s, 3 H), 3.72 (s, 3 H), 3.54-3.50 (m, 1 H), 3.48 (br s, 1 H), 2.49-2.45 (m, 3 H), 2.41-2.39 (m, 3 H), 1.65-1.59 (m, 1 H), 1.50-1.45 (m, 1 H), 1.42-1.36 (m, 1 H), 1.23-1.18 (m, 1 $\mathrm{H}) ;{ }^{13} \mathrm{C} \mathrm{NMR}\left(\mathrm{CDCl}_{3}, 125 \mathrm{MHz}\right) \delta 213.8,153.4,150.9,146.1,130.9,118.9,117.7,112.0,111.9$, 61.3, 55.9, 55.6, 41.4, 40.7, 35.7, 34.0, 27.2, 24.6; HRMS (EI) calcd for $\mathrm{C}_{18} \mathrm{H}_{22} \mathrm{O}_{3}\left(\mathrm{M}^{+}\right) \mathrm{m} / \mathrm{z}$ 286.1569, found 286.1569. 
7-(2,5-Dimethoxyphenyl)-2-methylhept-1-en-6-yn-3-ol (4). Following the procedure described for the synthesis of 2, alcohol 4 was prepared as a pale yellow oil in 90\% yield. IR (neat) 3430, 2936, 1652, 1498, $1047 \mathrm{~cm}^{-1}$; H NMR ( $\left.\mathrm{CDCl}_{3}, 500 \mathrm{MHz}\right) \delta 6.90$ (d, $\left.J=3.0 \mathrm{~Hz}, 1 \mathrm{H}\right)$, 6.78-6.74 (m, 2 H), 5.01 (s, 1 H), 4.86 (t, $J=1.5 \mathrm{~Hz}, 1 \mathrm{H}$ ), 4.29 (dt, $J=$ 7.5, $4.0 \mathrm{~Hz}, 1 \mathrm{H}$ ), 3.80 (s, $3 \mathrm{H}$ ), 3.72 (s, 3 H), 2.58-2.49 (m, 2 H), 2.39 (d, $J=2.5$ Hz, 1 H), 1.87-1.80 (m, 2 H), 1.74 (s, $3 \mathrm{H}) ;{ }^{13} \mathrm{C}$ NMR $\left(\mathrm{CDCl}_{3}, 125 \mathrm{MHz}\right) \delta$ 154.3, 153.0, 146.8, 118.1, 114.6, 113.2, 111.6, 110.9, 93.8, 77.2, 74.7, 56.2, 55.6, 33.7, 17.8, 16.2; HRMS (EI) calcd for $\mathrm{C}_{16} \mathrm{H}_{20} \mathrm{O}_{3}\left(\mathrm{M}^{+}\right) \mathrm{m} / \mathrm{z}$ 260.1413, found 260.1416.

1-Cyclohexenylpent-4-yn-1-ol (6). To a $-78^{\circ} \mathrm{C}$ solution of 1-iodocyclohexane (831 mg, 3.99 $\mathrm{mmol})$ in $15 \mathrm{~mL} \mathrm{Et}_{2} \mathrm{O}$ was added ${ }^{\mathrm{t}} \mathrm{BuLi}(1.7 \mathrm{M}$ in pentane, $4.7 \mathrm{~mL}, 8.0 \mathrm{mmol})$ dropwise. The resulting solution was stirred at $-78^{\circ} \mathrm{C}$ for $15 \mathrm{~min}$, then warmed to $0{ }^{\circ} \mathrm{C}$ and stirred for another $15 \mathrm{~min}$ at this temperature to destroy any excess ${ }^{t} \mathrm{BuLi}$. The solution was then cooled to $-78{ }^{\circ} \mathrm{C}$, followed by the dropwise addition of 5-(trimethylsilyl)pent-4-ynal (308 mg, $2.00 \mathrm{mmol}$ ) dissolved in $\mathrm{Et}_{2} \mathrm{O}(15 \mathrm{~mL})$. The reaction mixture was stirred thereafter for 20 min and then quenched by the addition of aqueous $\mathrm{NH}_{4} \mathrm{Cl}$. The layers were separated and the aqueous layer was extracted with $\mathrm{Et}_{2} \mathrm{O}$. The combined organic extracts were washed with brine, dried over $\mathrm{MgSO}_{4}$, filtered, and concentrated under reduced pressure. The residue was immediately dissolved in $10 \mathrm{~mL}$ THF, and TBAF (1.0 M in THF, $3.0 \mathrm{~mL}, 3.0 \mathrm{mmol}$ ) was added. The resulting mixture was stirred for $10 \mathrm{~min}$ at room temperature, followed by addition of water (3 $\mathrm{mL}$ ) to quench the reaction. The layers were separated and the aqueous layer was extracted with ether ( 2 x $20 \mathrm{~mL}$ ). The combined organic extracts were washed with brine, dried over $\mathrm{MgSO}_{4}$, filtered and concentrated under reduced pressure. The product was purified by column chromatography (15\% EtOAc in hexane) to give alcohol 6 as a colorless oil (295 mg, two steps, 90\%). IR (neat) 3308, 2927, 1558, 1051, $824 \mathrm{~cm}^{-1} ;{ }^{1} \mathrm{H}$ NMR $\left(\mathrm{CDCl}_{3}, 500 \mathrm{MHz}\right) \delta 5.69$ (s, $\left.1 \mathrm{H}\right)$, 4.10 (t, $J=7.0 \mathrm{~Hz}, 1 \mathrm{H}$ ), 2.26-2.21 (m, $2 \mathrm{H}), 2.07-2.00$ (m, $3 \mathrm{H}$ ), 1.96 (t, $J=2.5 \mathrm{~Hz}, 1 \mathrm{H}), 1.92-$ 1.86 (m, $1 \mathrm{H}), 1.77$ (dd, $J=13.0,6.5 \mathrm{~Hz}, 2 \mathrm{H}), 1.64-1.51$ (m, $5 \mathrm{H}) ;{ }^{13} \mathrm{C} \mathrm{NMR}\left(\mathrm{CDCl}_{3}, 125 \mathrm{MHz}\right)$ $\delta$ 139.2, 123.4, 84.2, 75.2, 68.5, 33.5, 24.9, 23.5, 22.6, 22.5, 15.0; HRMS (EI) calcd for $\mathrm{C}_{11} \mathrm{H}_{16} \mathrm{O}$ $\left(\mathrm{M}^{+}\right) \mathrm{m} / \mathrm{z}$ 164.1201, found 164.1202 . 
7-(2,5-Dimethoxyphenyl)hept-1-en-6-yn-3-ol (8). To a solution of aldehyde 1 (150 mg, 0.69 mmol) in $8 \mathrm{ml}$ of THF was added vinylmagnesium chloride (1.6 M in THF, $0.86 \mathrm{~mL}, 1.38$ mmol) at $-78^{\circ} \mathrm{C}$ and the resulting mixture was stirred for $15 \mathrm{~min}$. A solution of aqueous $\mathrm{NH}_{4} \mathrm{Cl}$ solution was then added to quench the reaction. The layers were separated and the aqueous layer was extracted with $\mathrm{Et}_{2} \mathrm{O}$. The combined organic extracts were washed with brine, dried over $\mathrm{MgSO}_{4}$, filtered and concentrated under reduced pressure. The crude product was purified by column chromatography (20\% EtOAc in hexane) to give alcohol 8 as a pale yellow oil (160 mg, 94\%). IR (neat) 3421, 2927, 1497, 1047, $824 \mathrm{~cm}^{-1} ;{ }^{1} \mathrm{H}$ NMR $\left(\mathrm{CDCl}_{3}, 500 \mathrm{MHz}\right) \delta 6.91$ (d, $J=$ 3.0 Hz, 1 H), 6.80-6.75 (m, 2 H), 5.94- 5.87 (m, 1 H), 5.32 (d, $J=17.0$ Hz, 1 H), 5.15 (d, $J=9.5$ Hz, 1 H), 4.39-4.35 (m, 1 H), 3.81 (s, 3H), 3.74 (m, 3 H), 2.65-2.52 (m, 2 H), 2.28 (br s, 1 H), 1.90-180 (m, $2 \mathrm{H}) ;{ }^{13} \mathrm{C}$ NMR $\left(\mathrm{CDCl}_{3}, 125 \mathrm{MHz}\right) \delta$ 154.4, 153.1, 140.4, 118.1, 114.8, 114.7, 113.2, 111.7, 93.7, 77.4, 72.1, 56.2, 55.7, 35.4, 16.0; HRMS (EI) calcd for $\mathrm{C}_{15} \mathrm{H}_{18} \mathrm{O}_{3}\left(\mathrm{M}^{+}\right) \mathrm{m} / \mathrm{z}$ 246.1256, found 246.1257 .

6-methylhept-6-en-4-ynal. To a solution of 6-methylhept-6-en-4-yn-1-ol (430 mg, 3.46 mmol) in DCM (35 mL) was added Dess-Martin periodinane $(2.20 \mathrm{~g}, 5.19 \mathrm{mmol})$ and the resulting mixture was stirred at room temperature for $12 \mathrm{~h}$. Aqueous solutions of sat. $\mathrm{Na}_{2} \mathrm{CO}_{3}(10 \mathrm{~mL})$ and sat. $\mathrm{Na}_{2} \mathrm{~S}_{2} \mathrm{O}_{2}(10 \mathrm{~mL})$ were then added and the heterogeneous mixture was stirred rapidly thereafter for $30 \mathrm{~min}$. The layers were separated and the aqueous layer was extracted with DCM (3x15 mL). The combined organic extracts were washed with brine, dried over $\mathrm{MgSO}_{4}$, filtered and concentrated under reduced pressure. The crude product was purified by column chromatography (10\% EtOAc in hexane) to give the desired aldehyde as a clear oil (285 mg, 67\%). IR (neat) 3067, 2923, 2865, 2740, 2316, 1772, $1733 \mathrm{~cm}^{-1}$; ${ }^{1} \mathrm{H}$ NMR $\left(\mathrm{CDCl}_{3}, 500 \mathrm{MHz}\right)$ $\delta 9.79(\mathrm{~s}, 1 \mathrm{H}), 5.18(\mathrm{~s}, 1 \mathrm{H}), 5.13-5.14(\mathrm{~m}, 1 \mathrm{H}), 2.64-2.69(\mathrm{~m}, 2 \mathrm{H}), 2.58-2.62(\mathrm{~m}, 1 \mathrm{H}), 1.82$ (br s, 3H); 201.0, 127.1, 121.2, 86.5, 42.4, 23.8, 12.5 ppm; ${ }^{13} \mathrm{C} \mathrm{NMR}\left(\mathrm{CDCl}_{3}, 125 \mathrm{MHz}\right) \delta 200.5$, 126.8, 121.1, 86.4, 82.8, 42.4, 23.5, 12.4 ppm; HRMS (EI) calcd for $\mathrm{C}_{8} \mathrm{H}_{10} \mathrm{O}\left(\mathrm{M}^{+}\right) \mathrm{m} / z$ 122.0732, found 122.0730 .

1-Cyclohexenyl-6-methylhept-6-en-4-yn-1-ol (10). A solution of ${ }^{t} \mathrm{BuLi}(6.20 \mathrm{~mL}, 1.7 \mathrm{M}$ in pentane, $10.6 \mathrm{mmol}$ ) was added dropwise to 1-bromocyclohexene (854 mg, $5.30 \mathrm{mmol}$ ) in diethyl ether at $-78{ }^{\circ} \mathrm{C}$. After $5 \mathrm{~min}$, the cooling bath was removed and the reaction mixture was 
allowed to warm to room temperature. After $30 \mathrm{~min}$ of stirring at this temperature the reaction mixture was re-cooled to $-78{ }^{\circ} \mathrm{C}$ wheupon a solution of 6-methylhept-6-en-4-ynal (210 mg, 1.72 mmol) in diethyl ether (10 mL) was added dropwise. After $30 \mathrm{~min}$, the reaction was quenched by the addition of $\mathrm{H}_{2} \mathrm{O}(3 \mathrm{~mL})$ and the mixture was allowed to warm to $0{ }^{\circ} \mathrm{C}$. Following the addition of more $\mathrm{H}_{2} \mathrm{O}(15 \mathrm{~mL})$, the layers were separated and the organic layer was extracted with diethyl ether (3x10 mL). The combined organic layers were dried over $\mathrm{MgSO}_{4}$, filtered and concentrated under reduced pressure. The crude product was purified by column chromatography on silica gel (70\% ethyl acetate/hexanes) to give $245 \mathrm{mg}$ (70\%) of the desired alcohol as a clear oil. IR (neat) 3350, 3094, 2925, 2857, 2836, 2225, 1652, 1448, $1049 \mathrm{~cm}^{-1} ;{ }^{1} \mathrm{H}$ NMR $\left(\mathrm{CDCl}_{3}, 500 \mathrm{MHz}\right) \delta$ 5.65-5.70 (m, 1H), 5.19 (br s, 1H), 5.12-5.14 (m, 1H), 4.08 (t, $J=5.87 \mathrm{~Hz}, 1 \mathrm{H}), 2.34$ (dt, $J=$ 7.23, $4.43 \mathrm{~Hz}, 2 \mathrm{H}), 1.88-2.05$ (m, 5H), 1.85 (br s, 3H), 1.75 (app. q, $J=6.83,2 \mathrm{H}$ ), 1.52-1.656 (m, 4H) ppm; ${ }^{13} \mathrm{C} \mathrm{NMR}\left(\mathrm{CDCl}_{3}, 125 \mathrm{MHz}\right) \delta$ 139.3, 127.2, 123.4, 120.5, 88.8, 82.2, 75.5, 33.8, 24.9, 23.8, 23.6, 22.6, 22.6, 15.9 ppm; HRMS (EI) calcd for $\mathrm{C}_{14} \mathrm{H}_{20} \mathrm{O}\left(\mathrm{M}^{+}\right) \mathrm{m} / \mathrm{z} 204.1514$, found 204.1515.

5-(Propan-2-ylidene)-3,4,4a,5,7,8-hexahydro-1H-benzo[7]annulen-6(2H)-one (11). Ketone 11 was prepared as a colorless oil in 45\% yield according to the general procedure described for the synthesis of 3 . IR (neat) 3012, 2925, 2859, 1700, 1652, 1558, 1456, $1050 \mathrm{~cm}^{-1}$; ${ }^{1} \mathrm{H}$ NMR $\left(\mathrm{CDCl}_{3}, 500 \mathrm{MHz}\right) \delta 5.49(\mathrm{dt}, J=9.3,1.70 \mathrm{~Hz}, 1 \mathrm{H}), 3.17$ (dd, $\left.J=12.45,3.66 \mathrm{~Hz}, 1 \mathrm{H}\right), 2.44-2.54$ (m, 2H), 2.14-2.20 (m, 1H), 1.90-2.02 (m, 2H), 1.72-1.78 (m, 2H), 1.73 (s, 3H), 1.64 (s, 3H), 1.57-1.1.63 (m, 2H), 1.34-1.44 (m, 1H), 1.16-1.27 (m, 2H) ppm; ${ }^{13} \mathrm{C} \mathrm{NMR}\left(\mathrm{CDCl}_{3}, 125 \mathrm{MHz}\right) \delta$ 213.0, 124.9, 139.9, 128.3, 121.1, 44.7, 43.6, 37.9, 34.8, 28.4, 26.6, 21.7, 21.5, 19.1 ppm; HRMS (EI) calcd for $\mathrm{C}_{14} \mathrm{H}_{20} \mathrm{O}\left(\mathrm{M}^{+}\right) \mathrm{m} / \mathrm{z} 204.1514$, found 204.1515 .

1-Cyclopent-4-yn-1-ol (12). Alcohol 12 was prepared as a colorless oil in 88\% yield according to the procedure described for the synthesis of $\mathbf{6}$. IR (neat) 3307, 2928, 1495, 1050, $824 \mathrm{~cm}^{-1} ;{ }^{1} \mathrm{H}$ NMR $\left(\mathrm{CDCl}_{3}, 500 \mathrm{MHz}\right) \delta 5.62(\mathrm{~s}, 1 \mathrm{H}), 4.39(\mathrm{t}, J=6.5 \mathrm{~Hz}, 1 \mathrm{H}), 2.34-2.26(\mathrm{~m}, 6 \mathrm{H}), 1.96(\mathrm{t}, J=$ $2.5 \mathrm{~Hz}, 1 \mathrm{H}), 1.91-1.73$ (m, $5 \mathrm{H}) ;{ }^{13} \mathrm{C} \mathrm{NMR}\left(\mathrm{CDCl}_{3}, 125 \mathrm{MHz}\right) \delta$ 146.3, 125.8, 84.1, 67.0, 68.6, 34.0, 32.1, 31.1, 23.3, 14.8; HRMS (EI) calcd for $\mathrm{C}_{10} \mathrm{H}_{14} \mathrm{O}\left(\mathrm{M}^{+}\right) \mathrm{m} / \mathrm{z}$ 150.1045, found 150.1049. 
1-Cyclopentenyl-5-phenylpent-4-yn-1-ol (14). Alcohol 14 was prepared as a colorless oil in 87\% yield according to the procedure described for the synthesis of $\mathbf{6}$. IR (neat) 3352, 2927, 1490, 1069, $824 \mathrm{~cm}^{-1}$; ${ }^{1} \mathrm{H}$ NMR $\left(\mathrm{CDCl}_{3}, 500 \mathrm{MHz}\right) \delta$ 7.43-7.40 (m, $\left.2 \mathrm{H}\right)$, 7.32-7.28 (m, $\left.3 \mathrm{H}\right)$, 5.69 (s, 1 H), 4.48 (t, $J=6.0$ Hz, 1 H), 2.53- 2.48 (m, 2 H), 2.39-2.33 (m, 4 H), 1.94-1.85 (m, 5 $\mathrm{H}) ;{ }^{13} \mathrm{C} \mathrm{NMR}\left(\mathrm{CDCl}_{3}, 125 \mathrm{MHz}\right) \delta 146.3,131.5(2 \mathrm{C}), 128.1(2 \mathrm{C}), 127.5,125.8,123.7,89.6,80.9$, 70.2, 34.3, 32.2, 31.2, 23.3, 15.8; HRMS (EI) calcd for $\mathrm{C}_{16} \mathrm{H}_{18} \mathrm{O}\left(\mathrm{M}^{+}\right) \mathrm{m} / \mathrm{z} 226.1358$, found 226.1358 .

1-Cyclohexenyl-5-(2,5-dimethoxyphenyl)pent-4-yn-1-ol (16). Alcohol 16 was prepared as a pale yellow oil in $87 \%$ yield according to the procedure described for the synthesis of 2 . IR (neat) 3446, 2926, 1496, 1049, $824 \mathrm{~cm}^{-1} ; \delta 6.91$ (d, $\left.J=2.0 \mathrm{~Hz}, 1 \mathrm{H}\right), 6.80-6.75$ (m, $\left.2 \mathrm{H}\right), 5.73$ (s, $1 \mathrm{H}), 4.19$ (t, $J=6.5 \mathrm{~Hz}, 1 \mathrm{H}), 3.82$ (s, $3 \mathrm{H}), 3.74$ (s, $3 \mathrm{H}), 2.54-2.48$ (m, $2 \mathrm{H}), 2.09-2.02$ (m, 4 H), 1.96-1.90 (m, $1 \mathrm{H}), 1.88$ (ddd, $J=7.0,1.5,1.5 \mathrm{~Hz}, 2 \mathrm{H}), 1.66-1.54$ (m, $4 \mathrm{H})$; ${ }^{13} \mathrm{C}$ NMR $\left(\mathrm{CDCl}_{3}, 125 \mathrm{MHz}\right) \delta 154.4,153.1,139.3,123.1,118.2,114.8,113.4,111.7,94.1,77.2,75.6,56.3$, 55.7, 33.8, 24.9, 23.7, 22.6, 22.6, 16.5; HRMS (EI) calcd for $\mathrm{C}_{19} \mathrm{H}_{24} \mathrm{O}_{3}\left(\mathrm{M}^{+}\right) \mathrm{m} / \mathrm{z} 300.1726$, found 300.1722 .

\section{(E)-10-(tert-Butyldimethylsilyloxy)-1-(2,5-dimethoxyphenyl)dec-6-en-1-yn-5-ol (18).}

Alcohol 18 was prepared as a pale yellow oil in 85\% yield according to the procedure described for the synthesis of 2. IR (neat) 3446, 2930, 1499, 1101, $835 \mathrm{~cm}^{-1} ;{ }^{1} \mathrm{H} \mathrm{NMR}\left(\mathrm{CDCl}_{3}, 500 \mathrm{MHz}\right)$ $\delta 6.90$ (d, $J=3.0$ Hz, 1 H), 6.79-6.74 (m, 2 H), 5.73-5.67(m, 1 H), 5.53-5.48 (m, 1 H), 4.30 (dd, $J=12.5,6.0 \mathrm{~Hz}, 1 \mathrm{H}), 3.80$ (s, $3 \mathrm{H}), 3.72$ (s, $3 \mathrm{H}), 3.60$ (t, $J=6.5$ Hz, 2 H), 2.61-2.48 (m, 2 H), 2.23 (s, 1 H), 2.10 (dd, $J=14.5,7.0$ Hz, 2 H), 1.85-1.76 (m, 2 H), 1.61-1.56 (m, 2 H), 0.88 (s, 9 $\mathrm{H}), 0.03$ (s, $6 \mathrm{H}) ;{ }^{13} \mathrm{C} \mathrm{NMR}\left(\mathrm{CDCl}_{3}, 125 \mathrm{MHz}\right) \delta 154.3,153.1,132.4,131.6,118.1,114.7,113.3$, 111.7, 93.8, 77.2, 71.9, 62.4, 56.2, 55.6, 35.8, 32.2, 28.4, 25.9, 18.2, 16.1, -5.4; HRMS (EI) calcd for $\mathrm{C}_{24} \mathrm{H}_{38} \mathrm{O}_{4} \mathrm{Si}\left(\mathrm{M}^{+}\right) \mathrm{m} / \mathrm{z}$ 418.2539, found 418.2537.

Ethyl 6-hydroxyoct-7-en-2-ynoate (20). To a $-78^{\circ} \mathrm{C}$ solution of ethyl 6-oxohex-2-ynoate (150 mg, $0.97 \mathrm{mmol}$ ) in $6 \mathrm{~mL}$ THF was added vinylmagnesium chloride (1.6 M in THF, 0.91mL, 1.45 mmol) and the resulting mixture was stirred at this temperature for an additional $15 \mathrm{~min}$. The reaction was then quenched by the addition of aqueous $\mathrm{NH}_{4} \mathrm{Cl}$ solution. Upon warming, the 
layers were separated and the aqueous layer was extracted with $\mathrm{Et}_{2} \mathrm{O}(2 \times 20 \mathrm{~mL})$. The combined organic extracts were washed with brine, dried over $\mathrm{MgSO}_{4}$, filtered, and concentrated under reduced pressure. The crude product was purified by column chromatography (20\% EtOAc in hexane) to give alcohol 20 as a pale yellow oil (150 mg, 85\%). IR (neat) 3422, 2929,1707, 1686, 1260, 1075, $824 \mathrm{~cm}^{-1}$; ${ }^{1} \mathrm{H}$ NMR $\left(\mathrm{CDCl}_{3}, 500 \mathrm{MHz}\right) \delta 5.83$ (ddd, $J=16.5,5.5,5.5 \mathrm{~Hz}, 1 \mathrm{H}$ ), 5.24 (d, $J=16.5 \mathrm{~Hz}, 1 \mathrm{H}), 5.11$ (d, $J=10.5 \mathrm{~Hz}, 1 \mathrm{H}), 4.18-4.14$ (m, $3 \mathrm{H}), 2.45-2.35$ (m, $3 \mathrm{H}$ ), 1.761.71 (m, $2 \mathrm{H}), 1.27$ (t, $J=7.5 \mathrm{~Hz}, 3 \mathrm{H}) ;{ }^{13} \mathrm{C}$ NMR $\left(\mathrm{CDCl}_{3}, 125 \mathrm{MHz}\right) \delta$ 153.7, 139.9, 115.3, 88.7, 73.2, 71.2, 61.7, 34.2, 14.7, 13.9; HRMS (EI) calcd for $\mathrm{C}_{10} \mathrm{H}_{14} \mathrm{O}_{3}\left(\mathrm{M}^{+}\right) \mathrm{m} / \mathrm{z}$ 182.0943, found 182.0943.

2-(2,5-Dimethoxyphenyl)-4-methylcyclohept-4-enone (5). Ketone 5 was prepared as a pale yellow oil in 73\% yield according to the general procedure described for the synthesis of $\mathbf{3}$. IR (neat) 2927, 1706, 1496, 1050, $824 \mathrm{~cm}^{-1} ;{ }^{1} \mathrm{H}$ NMR (CDCl $\left.3,500 \mathrm{MHz}\right) \delta 6.79-6.73$ (m, $\left.3 \mathrm{H}\right), 5.60$ (br s, 1 H), 4.32 (dd, $J=7.0,3.5$ Hz, 1 H), 3.77 (s, 3 H), 3.73 (s, 3 H), 3.01-2.95 (m, 1 H), 2.90 (t, $J=15.5 \mathrm{~Hz}, 1 \mathrm{H}), 2.65-2.57$ (m, $2 \mathrm{H}), 2.30-2.25$ (m, $2 \mathrm{H}), 1.77$ (s, $3 \mathrm{H}) ;{ }^{13} \mathrm{C} \mathrm{NMR}\left(\mathrm{CDCl}_{3}\right.$, $125 \mathrm{MHz}) \delta 212.7,153.6,150.7,136.2,130.9,123.2,115.6,111.7,111.4,55.9,55.6,50.5,42.3$, 35.7, 26.6, 24.1; HRMS (EI) calcd for $\mathrm{C}_{16} \mathrm{H}_{20} \mathrm{O}_{3}\left(\mathrm{M}^{+}\right) \mathrm{m} / \mathrm{z}$ 260.1413, found 260.1415 .

3,4,4a,5,7,8-Hexahydro-1 $\mathrm{H}$-benzo[7]annulen-6(2H)-one (7). Ketone 7 was prepared as a colorless oil in $77 \%$ yield according to the general procedure described for the synthesis of $\mathbf{3}$. IR (neat) 2926, 1706, 1495, 1050, $825 \mathrm{~cm}^{-1} ;{ }^{1} \mathrm{H} \mathrm{NMR}\left(\mathrm{CDCl}_{3}, 500 \mathrm{MHz}\right) \delta 5.56(\mathrm{t}, J=6.0 \mathrm{~Hz}, 1$ H), 2.84 (dd, $J=11.5,4.5$ Hz, 1 H), 2.73 (dd, $J=11.5,4.5$ Hz, 1 H), 2.53-2.44 (m, 3 H), 2.302.23 (m, 2 H), 2.18-2.14 (m, 1 H), 1.95-1.90 (m, 1 H), 1.79-1.73 (m, 3 H), 1.42-1.36 (m, 1 H), 1.28-1.20 (m, $2 \mathrm{H}) ;{ }^{13} \mathrm{C}$ NMR $\left(\mathrm{CDCl}_{3}, 125 \mathrm{MHz}\right) \delta$ 213.3, 143.7, 120.3, 47.1, 44.0, 40.8, 38.2, 36.1, 28.4, 26.5, 21.7; HRMS (EI) calcd for $\mathrm{C}_{11} \mathrm{H}_{16} \mathrm{O}\left(\mathrm{M}^{+}\right) \mathrm{m} / \mathrm{z}$ 164.1201, found 164.1203.

2-(2,5-Dimethoxyphenyl)cyclohept-4-enone (9). Ketone 9 was prepared as a colorless oil in $73 \%$ yield according to the general procedure described for the synthesis of 3. IR (neat) 2934, 1706, 1497, 1226, 1049, $823 \mathrm{~cm}^{-1} ;{ }^{1} \mathrm{H}$ NMR $\left(\mathrm{CDCl}_{3}, 500 \mathrm{MHz}\right) \delta$ 6.79-6.73 (m, $\left.3 \mathrm{H}\right)$, 5.85-5.77 (m, 2 H), 4.18 (dd, $J=11.5,3.5$ Hz, 1 H), 3.76 (s, 3 H), 3.72 (s, 3 H), 3.15 (ddd, $J$ = 11.5, 4.0, 4.0 Hz, $1 \mathrm{H}$ ), 2.93-2.86 (m, $1 \mathrm{H}), 2.66-2.54$ (m, $2 \mathrm{H}), 2.39-2.34$ (m, $2 \mathrm{H}) ;{ }^{13} \mathrm{C} \mathrm{NMR}\left(\mathrm{CDCl}_{3}, 125\right.$ 
MHz) $\delta$ 213.0, 153.6, 150.7, 131.1, 129.3, 128.5, 115.8, 111.9, 111.6, 55.9, 55.6, 52.6, 41.7, 30.3, 25.1; HRMS (EI) calcd for $\mathrm{C}_{15} \mathrm{H}_{18} \mathrm{O}_{3}\left(\mathrm{M}^{+}\right) \mathrm{m} / \mathrm{z}$ 246.1256, found 246.1256.

2,3,3a,4,6,7-Hexahydroazulen-5(1H)-one (13). Ketone 13 was prepared as a colorless oil in $72 \%$ yield according to the general procedure described for the synthesis of 3. IR (neat) 2937, 1706, 1495, 1650, 1049, $824 \mathrm{~cm}^{-1} ;{ }^{1} \mathrm{H}$ NMR $\left(\mathrm{CDCl}_{3}, 500 \mathrm{MHz}\right) \delta 5.62(\mathrm{dt}, J=6.0,2.5 \mathrm{~Hz}, 1 \mathrm{H})$, 2.89-2.84 (m, 1 H), 2.77-2.74 (m, 1 H), 2.60 (dd, $J=15.5,3.0$ Hz, 1 H), 2.45 (dd, $J=15,2.0$ Hz, 1 H), 2.38-2.32 (m, 4 H), 2.28-2.22 (m, 1 H), 2.03-1.97 (m, 1 H), 1.73-1.66 (m, 1 H), 1.57-1.49 (m, $1 \mathrm{H}), 1.36-1.28(\mathrm{~m}, 1 \mathrm{H}) ;{ }^{13} \mathrm{C}$ NMR $\left(\mathrm{CDCl}_{3}, 125 \mathrm{MHz}\right) \delta$ 213.1, 148.0, 119.2, 49.4, 42.3, 37.9, 35.1, 34.7, 25.5, 24.9; HRMS (EI) calcd for $\mathrm{C}_{10} \mathrm{H}_{14} \mathrm{O}\left(\mathrm{M}^{+}\right) \mathrm{m} / \mathrm{z}$ 150.1045, found 150.1046 .

(3aS*,4R*)-4-Phenyl-2,3,3a,4,6,7-hexahydroazulen-5(1H)-one (15). Ketone 15 was prepared as a yellow oil in 77\% yield according to the general procedure described for the synthesis of $\mathbf{3}$. IR (neat) 2928, 1700, 1495, 1049, 824 $\mathrm{cm}^{-1} ;{ }^{1} \mathrm{H} \mathrm{NMR}\left(\mathrm{CDCl}_{3}, 500 \mathrm{MHz}\right) \delta$ 7.26-7.23 (m, $2 \mathrm{H}$ ), 7.21-7.18 (m, 3 H), 5.51 (s, 1 H), 3.45 (d, $J=11.5$ Hz, 1 H), 3.31- 3.26 (m, 1 H), 3.23 (ddd, $J=$ 11.0, 5.5, 5.5 Hz, 1 H), 2.46-2.38 (m, 2 H), 2.36-2.32 (m, 3 H), 1.67- 1.60 (m, 1 H), 1.58-1.52 (m, $1 \mathrm{H}), 1.45-1.47$ (m, $1 \mathrm{H}), 1.22-1.15$ (m, $1 \mathrm{H}) ;{ }^{13} \mathrm{C} \mathrm{NMR}\left(\mathrm{CDCl}_{3}, 125 \mathrm{MHz}\right) \delta 211.8,146.1$, 138.6, 128.6 (2C), 128.3 (2C), 127.1, 119.0, 65.6, 42.2, 39.7, 35.9, 33.4, 28.0, 24.7; HRMS (EI) calcd for $\mathrm{C}_{16} \mathrm{H}_{18} \mathrm{O}\left(\mathrm{M}^{+}\right) \mathrm{m} / \mathrm{z} 226.1358$, found 226.1357 .

(4aS*,5R*)-5-(2,5-dimethoxyphenyl)-3,4,4a,5,7,8-hexahydro-1H-benzo[7]annulen-6(2H)-

one (17). Ketone 17 was prepared as a yellow oil in $82 \%$ yield according to the general procedure described for the synthesis of 3. IR (neat) 2928, 1700, 1495, 1049, $824 \mathrm{~cm}^{-1}$; ${ }^{1} \mathrm{H}$ NMR $\left(\mathrm{CDCl}_{3}, 500 \mathrm{MHz}\right) \delta 7.02(\mathrm{~d}, J=3.0 \mathrm{~Hz}, 1 \mathrm{H}), 6.78-6.73$ (m, $\left.2 \mathrm{H}\right), 5.61$ (dd, $J=9.0,4.5 \mathrm{~Hz}, 1$ H), 4.93 (d, $J=11.5 \mathrm{~Hz}, 1 \mathrm{H}), 3.79$ (s, 3 H), 3.73 (s, 3 H), 2.95-2.87 (m, 1 H), 2.73-2.60 (m, 2 H), 2.49 (t, $J=11.5$ Hz, 1 H), 2.21-2.18 (m, 1 H), 2.14-2.08 (m, 1 H), 1.99-1.94 (m, 1 H), 1.811.76 (m, $2 \mathrm{H}), 1.72$ (d, $J=13.0 \mathrm{~Hz}, 1 \mathrm{H}), 1.39-1.27$ (m, $2 \mathrm{H}), 1.21-1.12$ (m, $1 \mathrm{H}) ;{ }^{13} \mathrm{C}$ NMR (CDCl $3,125 \mathrm{MHz}) \delta$ 211.1, 153.6, 151.6, 144.4, 127.9, 119.6, 115.6, 111.4, 110.9, 56.1, 55.7, 49.7, 46.3, 45.6, 39.3, 33.8, 29.3, 26.7, 20.9; HRMS (EI) calcd for $\mathrm{C}_{19} \mathrm{H}_{24} \mathrm{O}_{3}\left(\mathrm{M}^{+}\right) \mathrm{m} / \mathrm{z} 300.1726$, found 300.1728 . 
$\left(2 R^{*}, 3 R^{*}\right)$-3-(3-(tert-Butyldimethylsilyloxy)propyl)-2-(2,5-dimethoxyphenyl)cyclohept-4-

enone (19). Ketone 19 was prepared as a yellow oil in 81\% yield according to the general procedure described for the synthesis of 3. IR (neat) 2928, 1700, 1496, 1050, $825 \mathrm{~cm}^{-1}$; ${ }^{1} \mathrm{H}$ NMR $\left(\mathrm{CDCl}_{3}, 500 \mathrm{MHz}\right) \delta 6.82(\mathrm{~d}, J=3.0 \mathrm{~Hz}, 1 \mathrm{H}), 6.78-6.72(\mathrm{~m}, 2 \mathrm{H}), 5.75-5.71(\mathrm{~m}, 1 \mathrm{H}), 5.65-5.62$ (m, 1 H), 4.09 (d, $J=11.0 \mathrm{~Hz}, 1 \mathrm{H}), 3.76$ (s, $3 \mathrm{H}$ ), 3.73 (s, $3 \mathrm{H}), 3.49$ (dt, $J=6.0,1.5 \mathrm{~Hz}, 2 \mathrm{H}$ ), 3.27-3.21 (m, 1 H), 3.13-3.08 (m, 1 H), 2.59-2.49 (m, 2 H), 2.43-2.37 (m, 1 H), 1.64-1.55 (m, 1 H), 1.48-1.39 (m, 1 H), 1.34-1.28 (m, 1 H), 1.22-1.13 (m, 1 H), 0.83 (s, 9 H), -0.03 (s, 3 H), 0.04 (s, $3 \mathrm{H}) ;{ }^{13} \mathrm{C} \mathrm{NMR}\left(\mathrm{CDCl}_{3}, 125 \mathrm{MHz}\right) \delta$ 212.3, 153.5, 151.3, 134.2, 129.0, 128.6, 116.6, 111.9, 111.7, 63.1, 57.4, 55.9, 55.6, 41.5, 38.5, 29.9, 29.7, 25.9, 25.3, 18.2, -5.4; HRMS (EI) calcd for $\mathrm{C}_{24} \mathrm{H}_{38} \mathrm{O}_{4} \mathrm{Si}\left(\mathrm{M}^{+}\right) \mathrm{m} / \mathrm{z}$ 418.2539, found 418.2536.

1-Cyclohexenyl-2,2-dimethylhex-4-yn-1-ol (22). Alcohol 22 was prepared as a pale yellow oil from 2,2-dimethylhex-4-ynal ${ }^{2}$ in $65 \%$ yield according to the procedure described for the synthesis of 2. IR (neat) 3448, 2929, 1653, 1136, $\left.1050 \mathrm{~cm}^{-1} ;{ }^{1} \mathrm{HNMR}_{(\mathrm{CDCl}}, 500 \mathrm{MHz}\right) \delta 5.64$ (s, 1 H), 3.86 (s, 1 H), 2.20 (ddd, $J=16.5,5.5,2.5$ Hz, 1 H), 2.14-2.10 (m, 1 H), 2.06-2.02 (m, 3 H), 1.98-1.95 (m, $1 \mathrm{H}), 1.81$ (d, $J=4.0 \mathrm{~Hz}, 1 \mathrm{H}), 1.80$ (t, $J=3.0 \mathrm{~Hz}, 3 \mathrm{H}), 1.65-1.60$ (m, $2 \mathrm{H})$, 1.54-1.50 (m, 2 H), 0.96 (s, $3 \mathrm{H}), 0.93$ (s, $3 \mathrm{H}) ;{ }^{13} \mathrm{CNMR}\left(\mathrm{CDCl}_{3}, 125 \mathrm{MHz}\right) \delta 138.7,125.3,82.1$, 77.6, 77.2, 38.5, 30.1, 26.9, 25.1, 24.2, 23.1, 22.9, 22.6, 3.5; HRMS (EI) calcd for $\mathrm{C}_{14} \mathrm{H}_{22} \mathrm{O}\left(\mathrm{M}^{+}\right)$ $\mathrm{m} / \mathrm{z}$ 206.1671, found 206.1672 .

5,8,8-trimethyl-3,4,4a,5,7,8-hexahydro-1H-benzo[7]annulen-6(2H)-one (23). Ketone 23 was prepared as a colorless oil in 60\% yield according to the general procedure described for the synthesis of 3. IR (neat) 2930, 1700, 1652, 1456, 1049, $825 \mathrm{~cm}^{-1} ;{ }^{1} \mathrm{HNMR}\left(\mathrm{CDCl}_{3}, 500 \mathrm{MHz}\right) \delta$ 5.64 (s, 1 H), 5.13 (s, 0.5 H), 3.16-3.11 (m, 1 H), 2.83 (d, J = 12.5 Hz, 0.5 H), 2.62-2.59 (m, 0.5 H), 2.54 (dd, $J=33.0,16.5 \mathrm{~Hz}, 1 \mathrm{H}$ ), 2.41 (dd, $J=5.0,2.5 \mathrm{~Hz}, 0.5 \mathrm{H}$ ), 2.29-2.22 (m, $1.5 \mathrm{H}$ ), 2.20-2.10 (m, 2.5 H), 2.04-1.98 (m, 1 H), 1.96-1.90 (m, 0.5 H), 1.86- 1.75 (m, 4.5 H), 1.68-1.62 (m, 1.5 H), 1.40-1.34 (m, 3 H), 1.20 (s, 3 H), 1.17 (s, 1.5 H), 1.11 (d, J = 6.5 Hz, 1.5 H), 1.10 (s, $1.5 \mathrm{H}), 1.07$ (d, $J=6.5 \mathrm{~Hz}, 3 \mathrm{H}), 1.04$ (s, $3 \mathrm{H}) ;{ }^{13} \mathrm{CNMR}\left(\mathrm{CDCl}_{3}, 125 \mathrm{MHz}\right) \delta$ 213.6, 212.8, 142.1, 139.5, 131.7, 130.6, 56.5, 53.4, 51.3, 48.3, 46.3, 43.9, 39.6, 36.5, 34.5, 34.2, 33.7, 32.3,

\footnotetext{
${ }^{2}$ Cresson, P.; Corbier, J. C. R. Hebd. Seances Acad. Sci. Ser. C 1969, 268, 1614.
} 
32.3, 29.7, 29.3, 29.2, 28.8, 26.4, 25.6, 23.6, 15.6, 13.4; HRMS (EI) calcd for $\mathrm{C}_{14} \mathrm{H}_{22} \mathrm{O}\left(\mathrm{M}^{+}\right) \mathrm{m} / \mathrm{z}$ 206.1671, found 206.1671. 